\title{
ANNALS Of THE NEW YORK ACADEMY OF SCIINCES
}

This unedited manuscript has been submitted for publication in the Annals of the NYAS.This paper has not been copyedited.

\section{Laterality in the gestural communication of wild chimpanzees}

\begin{tabular}{|r|l|}
\hline Journal: & Annals of the New York Academy of Sciences \\
\hline Manuscript ID: & annals-1620-006 \\
\hline Manuscript Type: & Other papers \\
\hline Date Submitted by the Author: & $03-$ Oct-2012 \\
\hline Complete List of Authors: & $\begin{array}{l}\text { Hobaiter, Catherine; University of St Andrews, Psychology \& Neuroscience } \\
\text { Byrne, Richard; University of St Andrews, Psychology \& Neuroscience }\end{array}$ \\
\hline Keywords: & handedness, laterality, communication, chimpanzee, gesture \\
\hline
\end{tabular}


1 Laterality in the gestural communication of wild chimpanzees 2

3 Catherine Hobaiter and Richard W. Byrne

4

5 Centre for Social Learning and Cognitive Evolution and Scottish Primate Research

6 Group, School of Psychology, University of St Andrews, St Andrews, KY16 9JP,

$7 \quad$ Scotland

8

9

10

11

12

13

14

15

16

17

18

19

20

21

22 Correspondence: R W Byrne, School of Psychology and Neuroscience, University of

23 St Andrews, St Andrews, Fife, KY16 9JP, Scotland. E-mail: rwb@st-andrews.ac.uk 


\section{Keywords}

25 chimpanzee; gesture; handedness; laterality; communication

27 Running header

28 Laterality in chimpanzee gestural communication

30 Abstract

31 We examined hand preference in the intentional gestural communication of wild

32 chimpanzees in the Budongo forest, Uganda. Individuals showed a tendency to be

33 lateralized; on average, their absolute bias was around 0.25 . Lateralization was

34 incomplete even in individuals with major manual disabilities. Where individuals had a

35 stronger preference, this was more often towards the right hand; moreover, as age

36 increased, the direction (but not the extent) of hand preference shifted towards the right.

37 While the gestural repertoire as a whole was largely employed ambilateraly, object-

38 manipulation gestures showed a strong right-hand bias.

39

40

41

42

43 
47 Analyses of hand preference in great apes have focused disproportionately on food-

48 related manipulation: for instance, picking up food, ${ }^{1,2}$ getting food out of containers, ${ }^{3,4}$

49 processing natural foods, ${ }^{5,6}$ tool use while foraging, ${ }^{1,7,8}$ and begging for food; ${ }^{9-11}$

50 however, see Marchant and McGrew ${ }^{12,13}$ for a broad analysis across limb functions. In

51 chimpanzees, captive studies have often reported strong individual and population level

52 right-hand biases, ${ }^{3,9,11}$ whereas studies conducted in wild populations largely report

53 ambilateral preferences in most manual tasks, with the exception of tool use. Tool use

54 appears to be highly lateralized in each individual but in no consistent direction in the

55 population. ${ }^{8,12}$ In the light of recent evidence from human studies, that hand preference

56 can vary markedly within individuals depending on the task in hand, ${ }^{14}$ it has become

57 increasingly important to examine great ape hand preferences in contexts other than

58 feeding, ideally in wild populations living under ecologically relevant conditions.

59 In our species, right-handedness and left-hemisphere laterality for language have

60 long been considered related, making studies of manual laterality in great ape

61 communication an obvious starting point. Great apes have a rich, elaborate repertoire of

62 gestures that they use in an intentional manner to communicate about specific goals to

63 other individuals. ${ }^{15-20}$ Recent studies of gestural communication in captive chimpanzees

64 have reported both individual and population level right-handedness, ${ }^{9,21}$ with an increase

65 in right-handedness when gestures are produced together with vocalizations. ${ }^{11}$ However,

66 to date, work on hand use in gestural communication has been limited to captive groups,

67 in particular to the use of gestures in begging and pointing for food; moreover, the

68 strongest effects were found in individuals with a history of human rearing. ${ }^{9,11}$ 
69 We recently conducted the first systematic study of gestural communication in a

70 wild community of chimpanzees. This presented us with the opportunity to examine hand

71 preferences in a very large database of gestures, produced across a full range of

72 situational contexts, by all ages and sexes, and under ecologically relevant conditions.

\section{Method}

76 We define gestures as discrete, mechanically ineffective physical movements of the

77 whole body, limbs and/or head, used in intentional communication (i.e. directed to a

78 specific audience and towards a specific goal). Chimpanzees employ a repertoire of at

79 least 66 gesture types in their communication. We took it that a gesture was being used

80 intentionally if it (or a sequence of gestures separated by $<1 \mathrm{sec}$ ) was accompanied by one

81 or more of the following: checking of the recipient's state of attention, waiting for a

82 response and, if none, then showing persistence or elaboration in further gesturing. (See

83 Hobaiter \& Byrne, $2011^{20}$ for a full description of the repertoire and the criteria for

84 intentional use.) A number of gesture types involve actions that would not easily reveal

85 any lateral bias, for example: Clap (both palms brought together with audible contact),

86 Pirouette (signaler spins on their vertical axis) and Present-sexual (signaler approaches

87 backwards, exposing swelling or anus to recipient). We excluded these gestures, and

88 restricted our analyses to gestures of the hand and arms.

89

$90 \quad$ Subjects 
91 At the start of data collection in October 2007, the Sonso study community of

92 chimpanzees consisted of 81 named individuals. Following Reynolds (2005), ${ }^{22}$ we

93 defined age groups as follows: infants (0-4yrs11mnths), juveniles (5yrs-9yrs11mnths),

94 sub-adults (10yrs-13yrs11mnths $\$ / 14 y r s 11$ mnthsơ) and adults (14yrs $\$ / 15 y$ rso'and over).

95 Using these categories, the initial group composition was 32 adults ( 7 males and 25

96 females), 16 sub-adults (10 males and 6 females), 15 juveniles (6 males and 9 females)

97 and 18 infants ( 3 males and 15 females). Over the course of the 22 -month study, there

98 were 10 deaths or long-term disappearances, 6 immigrations and 5 births, leaving the

99 final total at 82.

100 A number of Sonso chimpanzees suffer from injuries caused by snare traps left in

101 the forest by bush-meat hunters from the local villages. In some cases the snare traps

102 sever tendons resulting in paralysis and in particularly severe cases may cause amputation

103 of the limb. The limitations of individual chimpanzees were well known and data from

104 individuals missing limbs or with damage to the whole hand or foot were examined

105 separately.

106

107 Procedure

108 Observations were made on chimpanzees within the Sonso community during three field

109 periods between October 2007 and August 2009 (October 2007-March 2008; June 2008-

110 January 2009; May 2009-August 2009). We employed focal behaviour sampling

111 (Altman, 1974), ${ }^{23}$ and filmed all instances of intentional gestural communication; in each

112 instance the data recorded included the signaler, recipient, gesture type, and limb(s) used;

113 for a detailed method and analysis protocol see Hobaiter \& Byrne 2011. ${ }^{20}$ 
115 Analysis

116 In any analysis of laterality it is important to ensure statistical independence in the data,

117 and each datum must represent a choice of limb unaffected by external influences from

118 the physical or social environment. For full details of the restrictions applied to the data

119 set to ensure independence, see ESM: Independence in the data.

120 To measure the direction of hand preference, both for individual chimpanzees and

121 within particular gesture types, we used the hand preference index (HI). HI is calculated

122 as $(\mathrm{R}-\mathrm{L}) / \mathrm{N}$, where $\mathrm{R}=$ frequency of right-hand use, $\mathrm{L}=$ frequency of left-hand use and

$123 \mathrm{~N}=$ total use. The index varies between -1.0 indicating complete left-hand use, through 0.0

124 (no preference), to +1.0 indicating compete right-hand use. Because the data are likely to

125 include a different balance of use among the potential set of gesture types between one

126 individual and another, and some gesture types may always be more lateralized than

127 others, we needed to normalize the data to avoid confounding these effects. We dealt

128 separately with (a) any possible effect of gesture differences in laterality upon individual

129 chimpanzees' laterality estimates; and (b) any possible effect of individual differences in

130 laterality upon laterality estimates for gesture types. Thus, we first calculated an

131 individual's HI for each gesture type, and then calculated the individual's mean HI across

132 gesture types. Similarly, we first calculated a gesture type's HI for each individual who

133 contributed data, and then calculated the gesture type's mean HI across individuals.

134 To measure the strength of hand preference, irrespective of direction, we used the

135 absolute hand preference index (ABS HI), calculated as ABS $\mathrm{HI}=\sqrt{ }\left(\mathrm{HI}^{2}\right)$. This varies

136 from 0.0 (no preference) to +1.0 (complete hand preference in either direction). As with 
137 the direction of hand preference, we calculated an individual's ABS HI after averaging

138 across gesture types, and for gesture types after averaging across individuals.

139 In order to test whether or not individuals or individual gesture types were

140 significantly lateralized in either direction we employed Goodness of Fit tests. This

141 required us to pool an individual's data across gesture types (and across individuals in the

142 case of gesture types), which runs the risk that pseudo replication may bias the findings;

143 the results are discussed with this in mind. Goodness of Fit tests were only applied to

144 individuals or gesture types that matched the requirement of a minimum expected 5-cases

145 in each cell. In practice, as the null hypothesis was a 50/50 distribution between left and

146 right hand use, this restricted the analyses to individuals or individual gesture types with

14710 or more gesture instances. Then, where the data were sufficiently homogeneous, a

148 pooled Goodness of Fit test was used to verify whether or not generalizations, that

149 appeared possible from analyses of individual chimpanzees or individual gesture type,

150 were significant when examined at a broader level of analysis. For example, we

151 compared all object manipulation gestures with all non-object manipulation gestures, and

152 male chimpanzees with female chimpanzees. All means are shown with standard

153 deviation; all statistical tests are 2-tailed.

\section{Results}

157 We recorded a total of 5026 gesture instances produced with concurrent evidence of

158 intentional usage, distributed across 66 gesture types. When restricted to gestures suitable

159 for examining any laterality effects, this reduced to 1274 instances across 20 gesture 
160 types: our analyses are all based on this sample, to which 54 individuals contributed data

161 (individual range=1-191 gestures, 1-15 gesture types).

162

163 Do individuals show a hand preference when gesturing?

164 Absolute hand preference strengths (ABS HI) ranged from 0.0 to 1.0: from no bias to

165 complete hand preference. (To avoid pseudo replication, we averaged the hand preference

166 scores for each of the gesture types a chimpanzee used.). For the population, the mean

167 ABS HI was $0.38 \pm 0.32(\mathrm{n}=54)$. However, as can be seen in Figure 1, the only individuals

168 that showed either zero or complete hand preference were those with less than four

169 gesture instances, and individual variation in hand preference appeared very high with

170 small samples. When we accordingly excluded individuals with fewer than 20 gesture

171 instances the range of individual preference decreased, to $0.02-0.67(n=21)$, and the

172 population mean ABS HI became $0.25 \pm 0.15$. An alternative way of estimating the true

173 degree of lateral bias in this population is the mean, weighted by the number of gesture

174 cases per individual, which gives an ABS HI of 0.26 .

175

176 Are lateralized hand preferences in one particular direction?

177 Hand preference scores (HI) range from -1.0 to 1.0 (to avoid pseudoreplication, we

178 averaged the hand preference scores for each of the gesture types a chimpanzee used.)

179 For the population, the mean ABS HI was $0.15 \pm 0.48(\mathrm{n}=54)$. However, once again,

180 individuals with very low numbers of gestures produce spuriously extreme hand

181 preference scores (see Figure 2). If we consider only individuals with more than 20

182 instances of gesture use contributing to their individual hand preference index $(n=21), 9$ 
183 had a left-hand preference and 12 a right-hand preference (range of individual preference

$184-0.31-0.67$; population mean $0.10 \pm 0.28$ ). Of these, only 5 were significantly lateralized,

185 four to the right (Janet: $\mathrm{n}=28, \mathrm{~g}=5.31, \mathrm{df}=1 \mathrm{p}=0.02$; Hawa: $\mathrm{n}=29, \mathrm{~g}=6.04, \mathrm{df}=1, \mathrm{p}=0.01$;

186 Zefa: $n=47, g=7.91, d f=1, p=0.005$; Nick: $n=85, g=20.63, d f=1, p<0.0001)$ and one to the

187 left (Zed: $\mathrm{n}=66, \mathrm{~g}=4.97, \mathrm{df}=1, \mathrm{p}-0.04)$; the group as a whole was too heterogeneous to

188 combine (heterogeneity goodness of fit $\mathrm{G}: \mathrm{g}=55.55, \mathrm{df}=20, \mathrm{p}=0.00003$ ). Eleven of the

189 individuals with $20+$ instances of gesture use had hand indices of $>0.25$ or $<-0.25$, i.e.

190 showed clear lateralization. Among this more lateralized group, two individuals showed a

191 left-hand preference (HI range -0.3 - -0.36) and eight showed a right-hand preference (HI

192 range $0.26-0.67$ ); this difference was not significant (Exact binomial test two-tailed,

$193 \mathrm{n}=10, \mathrm{p}=0.109)$.

194

195 Is there an effect of age on individual hand preference

196 For this analysis, individuals were assigned to four age groups: infants, juveniles,

197 subadults and adults. As the study was conducted across 3 years, individuals frequently

198 contributed data to more than one age-group, so the total number of 'individuals'

199 included in age related statistics $(n=114)$ was larger than the actual number of

200 chimpanzees in the population $(n=54)$, and the sample size for each 'individual' was

201 smaller than in other analyses. In this case, employing our previous restriction to

202 individuals with $20+$ gestures would eliminate the majority of individuals (from $n=114$ to

$203 \mathrm{n}=15)$, so we relaxed the criterion to include individuals with $10+$ gestures $(\mathrm{n}=38)$.

204 We found a significant effect of age on the direction of hand preference (One-way

205 Anova: $\mathrm{F}=3.16, \mathrm{df}=3,34, \mathrm{p}=0.037$ ), with individuals becoming more right-handed with 
206 age (Figure 3). There was no effect of age on the strength of hand preference (One-way

207 Anova: $\mathrm{F}=1.34, \mathrm{df}=3,34, \mathrm{p}=0.261)$.

208

209 Do snare injuries determine hand choice?

210 We examined the gesturing of 8 individuals with major snare injuries (hand amputated or

211 paralysed). Six of the snare-injured chimpanzees preferred their healthy hand; one

212 preferred the snared-hand, but only 2 cases of gesture use were recorded; and one

213 individual had severe snare-injuries to both hands. As a group the snare-injured

214 individuals were more lateralized than healthy chimpanzees (healthy group: $\mathrm{n}=21$, mean

215 ABS HI $=0.25 \pm 0.15$, snare-injured group: $\mathrm{n}=8$ mean $\mathrm{ABS} H \mathrm{H}=0.68 \pm 0.32$, $\mathrm{t}$-test: $\mathrm{t}=5.07$,

$216 \mathrm{df}=27 \mathrm{p}<0.0001)$. However, injured individuals varied greatly in their degree of hand

217 preference (ABS HI snared individuals: range=0.12-1.0). Only 2 of the 4 individuals

218 suitable for statistical testing with a binomial test (gesture cases $n>10$, individual data

219 pooled across gesture types) were significantly lateralized (Zig: $n=35, p=0.04$; Kana:

$220 \mathrm{n}=20, \mathrm{p}<0.0001)$, both in the direction of the less injured hand.

221

222 Does lateralization vary among gesture types?

223 Within the 20 gesture types suitable for analysis of lateralization, strength of hand

224 preference scores (ABS HI) for each type ranged from 0.0 to 1.0 , with mean $0.38 \pm 0.32$.

225 (To avoid pseudo replication, we averaged, for each gesture type, the scores of each

226 chimpanzee who contributed to the index.) However, as with the ABS HI scores for

227 individual chimpanzees (Figure 1), variation in the ABS HI scores of gesture types

228 decreased with an increase in the number of gesture instances. If analysis is restricted to 
229 gesture types with $20+$ instances of the gesture type $(n=8)$, the ABS HI range is $0.01-0.32$ 230 and the mean ABS HI is $0.20 \pm 0.11$.

231 Within the repertoire we found no clear direction in hand bias: 10 gestures had HI 232 index scores of less than zero, indicating some left-hand preference, and 10 above zero, 233 indicating right-hand preference. The mean HI index for all gestures studied was 0.04 $234 \pm 0.50(\mathrm{n}=20$; see Figure 4); when gestures with fewer than 20 cases were eliminated, this 235 rose to $0.17 \pm 0.15(\mathrm{n}=8)$.

236 While pooling individual data runs the risk of introducing pseudo-replication, we

237 felt that given the low levels of hand preference within the population this might be 238 worthwhile for investigating hand preference across gesture types. Twelve gesture types 239 had 10 or more instances of use (after pooling across all individuals) and could be tested 240 for hand bias with individual goodness of fit tests. Five of these showed a bias, one to the 241 left (Arm shake: $\mathrm{n}=11 \mathrm{~g}=4.82, \mathrm{df}=1 \mathrm{p}=0.028$ ), four to the right (Big Loud Scratch: $\mathrm{n}=222$ $242 \mathrm{~g}=4.63 \mathrm{df}=1 \mathrm{p}=0.031$; Object Shake: $\mathrm{n}=261 \mathrm{~g}=5.85 \mathrm{df}=1 \mathrm{p}=0.016$; Object move: $\mathrm{n}=103$ $243 \mathrm{~g}=6.13 \mathrm{df}=1 \mathrm{p}=0.013$; Hand fling: $\mathrm{n}=44 \mathrm{~g}=7.58 \mathrm{df}=1 \mathrm{p}=0.006$ ); all other gestures were 244 non-significant (Slap object with object, Punch object/ground, Arm swing, Slap object,

245 Reach, Leaf clipping, Arm raise). The variation across gesture types was too

246 heterogeneous to pool into a single repertoire score (heterogeneity goodness of fit

$247 \mathrm{G}=21.04, \mathrm{df}=11, \mathrm{p}=0.033)$, indicating that the use of different gesture types did not fit a

248 single pattern of hand bias. However the fact that two right-biased gestures involved

249 object use led us to carry out an additional analysis

250 We investigated whether or not object manipulation (OM) was a significant factor 251 by separating OM gesture types from non-object manipulation (NOM) gesture types. OM 
252 gestures were found to be sufficiently homogenous to combine, and the combined OM set

253 showed a significant right-handed bias ( $\mathrm{OM}$ total $\mathrm{G}=11.98, \mathrm{df}=2, \mathrm{p}=0.003$; pooled

$254 \mathrm{G}=11.31, \mathrm{df}=1, \mathrm{p}=0.0008$; heterogeneity $\mathrm{G}=0.67, \mathrm{df}=1, \mathrm{p}=0.414)$. NOM gestures were

255 also found to be sufficiently homogenous to combine, but in this case the combined NOM

256 set did not have a significant hand bias $(\mathrm{NOM}$ total $\mathrm{G}=19.54, \mathrm{df}=10, \mathrm{p}=0.034$; pooled

$257 \mathrm{G}=2.85, \mathrm{df}=1, \mathrm{p}=0.091$; heterogeneity $\mathrm{G}=16.69, \mathrm{df}=9, \mathrm{p}=0.054)$.

258

259 Discussion

260 Communicative gestures of wild chimpanzees at Budongo show very flexible hand use:

261 none of our subjects employed a single hand exclusively, and estimates of the strength of

262 hand preferences decreased with increasing amounts of data. Nevertheless, none of these

263 chimpanzees displayed perfect ambilaterality; there were consistent hand preferences

264 even in individuals with extremely large sample sizes; and the community as a whole

265 showed a slight right-hand bias.

266 If one requires that an individual's hand use be significantly lateralized to be

267 classified as a preference, then our findings correspond to Level 1 in McGrew and

268 Marchant's suggested framework 'most individuals in a group (or deme or species) are

269 ambipreferent and only a minority of individuals are lateralised to either side to varying

270 degrees'. ${ }^{13}$ Perhaps one of the most striking examples of incomplete lateralization is seen

271 in the snare-injured group of chimpanzees: although they had more pronounced hand

272 preferences than healthy chimpanzees, individuals persisted in gesturing at times with

273 their injured hand even in the face of massive physical deformity (e.g. complete

274 amputation of one hand). 
275 A possible explanation for this striking flexibility is that there is an environmental

276 benefit for ambilaterality in chimpanzee gestural communication; in other words, being

277 able to use either hand confers some advantage to a communicating individual. In termite

278 fishing complete lateralization was found to confer a small but significant advantage,

279 with $100 \%$ handed individuals more efficient in gathering termites, ${ }^{8}$ this might explain

280 the tendency towards lateralization in chimpanzee tool-use. The circumstances are

281 different for gesturing, however. Feeding chimpanzees are rarely engaged in other

282 activities, in marked contrast to gesturing chimpanzees who may well be grooming,

283 travelling, or playing at the same time. In addition, tool use usually occurs on the ground,

284 in open areas, whereas communication may occur anywhere: while hanging from a

285 climber, or travelling through dense undergrowth. Under these conditions it may be that

286 the ability to communicate with either hand - and, by doing so, to avoid having to stop

287 any concurrent activity or locomotion - represents sufficient advantage in maintaining

288 flexible use of either hand, even in the face of massive physical pressures such as

289 permanent injury. Testable predictions of this theory include (a) an individual's manual

290 lateralization should decrease when forced to operate in more difficult locations, for

291 example when using tools to break into arboreal beehives; (b) an individual's gestural

292 lateralization should increase in less complex environments with no other concurrent

293 activities, for example in captivity.

294 Despite the strong evidence for ambilateral hand use in gestural communication in

295 the wild Sonso chimpanzees, it is difficult to completely dismiss the pattern of small but

296 consistently right-handed biases we have found. More individuals favoured their right-

297 hand to some extent, whether we consider all individuals, only individuals with more than 
29820 cases of gesture use, or only individuals with stronger hand preferences. Mean

299 population hand preference, after correcting for any bias from either individuals or

300 gesture types, was to the right. Individuals become more right handed with age. The one

301 gesture class that showed significantly lateralization, the object manipulation gestures,

302 was lateralized to the right.

303 Our finding that right-hand use increases with age supports the similar findings

304 from captivity ${ }^{9}$ and suggests that this is not, as has been suggested, ${ }^{13}$ simply an effect of

305 human enculturation or exposure to a human designed environment.

306 Perhaps our most interesting finding is that of the striking right hand bias in object

307 manipulation gestures, which highlights the potential task-specificity of hand preferences.

308 Although several (less lateralized) gestures involve the use of an object or the ground as $a$

309 substrate (for example, object slaps or stomps), in the case of the (lateralized) Object

310 shake or Object move gestures, the object is actively manipulated. In captive gorillas,

311 hand preferences for uni-manual actions have been found to be affected by target

312 animacy, with inanimate targets eliciting increased right-hand use. ${ }^{24}$ Those authors

313 suggested that ape brain structures involved in object manipulations, such as tool use,

314 may have served as a precursor to those involved in language processing, so that a pre-

315 existing bias to left-brain processing led to the left-lateralization of language. Data from

316 wild gorillas are consistent this suggestion. The hierarchically-organized food processing

317 skills of wild gorillas have been noted as 'syntactically' structured, like a phrase-structure

318 grammar. $^{6}$ And several of these food-processing routines showed significant right-

319 handedness ${ }^{5}$. Our findings on chimpanzee gesture, however, suggest an alternative or 
320 additional explanation for human right-handedness: that language might have been

321 'scaffolded' on a primitive substrate for intentional communication in great ape gesture.

322

323

324 Acknowledgements

325 We would particularly like to thank A. Meguerditchian and W.D. Hopkins for their

326 enthusiastic encouragement in considering the issue of hand preference in our data set.

327 We thank all the staff of the Budongo Conservation Field Station, and the BCFS project's

328 founder Vernon Reynolds and its current scientific director Klaus Zuberbühler for

329 allowing us to work at the site. For permission to work in Uganda we thank the Uganda

330 National Council for Science and Technology, the President's Office, the Uganda

331 Wildlife Authority and the National Forestry Authority. Fieldwork of $\mathrm{CH}$ was generously

332 supported by grants from the Wenner-Gren Foundation and the Russell Trust.

\section{References}

336 1. Sugiyama, Y., Fushimi, T., Sakura, O. \& Matsuzawa, T. 1993. Hand preference and

337 tool use in wild chimpanzees. Primates 34(2): 151-159.

338

339 2. Peters, H.H. \& Rogers, L.J. 2008. Limb use and preferences in wild orang-utans during

340 feeding and locomotor behavior. Am. J. Primatol. 70: 261-270. 
342 3. Hopkins, W.D. 1995. Hand preferences for a coordinated bimanual task in 110

343 chimpanzees (Pan troglodytes): Cross-sectional analysis. J. Comp. Psychol. 109(3): 291-

344297.

345

346 4. Braccini, S., Lambeth, S., Schapiro, S. \& Fitch, W. T. 2010. Bipedal tool use

347 strengthens chimpanzee hand preferences. J. Hum. Evol. 58: 234-241.

348

349 5. Byrne, R.W. \& Byrne, J.M. 1991. Hand preferences in the skilled gathering tasks of

350 mountain gorillas (Gorilla g. beringei). Cortex 27: 521-546.

351

352 6. Byrne, R.W. \& Byrne, J.M. 1993. Complex leaf-gathering skills of mountain gorillas

353 (Gorilla g. beringei): Variability and standardization. Am. J. Primatol. 31: 241-261.

355 7. Boesch, C. 1991. Handedness in wild chimpanzees. Int. J. Primatol. 12(6): 541-558.

357 8. McGrew, W.C. \& Marchant, L.F. 1999. Laterality of hand use pays off in foraging

358 success for wild chimpanzees. Primates 40(3): 509-513.

359

360 9. Hopkins, W.D. \& Leavens, D.A. 1998. Hand use and gestural communication in

361 chimpanzees (Pan troglodytes). J. Comp. Psychol. 112(1): 95-99. 
363 10. Hopkins, W.D. \& Wesley, M.J. 2002. Gestural communication in chimpanzees (Pan

364 troglodytes): The influence of experimenter position on gesture type and hand preference.

365 Laterality 7(1): 19-30.

366

367 11. Hopkins, W.D. \& Cantero, M. 2003. From hand to mouth in the evolution of

368 language: the influence of vocal behavior on lateralized hand use in manual gestures by

369 chimpanzees (Pan troglodytes). Developmental Sci. 6(1): 55-61.

370

371 12. Marchant, L.F. \& McGrew, W.C. 1996. Laterality of limb function in wild

372 chimpanzees of Gombe National Park: comprehensive study of spontaneous activities. $J$.

373 Hum. Evol. 30: 427-443.

374

375 13. McGrew, W.C. \& Marchant, L.F. 2001. Ethological study of manual laterality in the

376 chimpanzees of the Mahale mountains, Tanzania. Behaviour 138(3): 329-358.

378 14. Rigal, R. A. 1992. Which handedness: Preference or performance? Percept. Motor

379 Skill. 75: 851-866.

380

381 15. Tomasello, M., George, B., Kruger, A., Farrar, J. \& Evans, E. 1985. The development

382 of gestural communication in young chimpanzees. J. Hum. Evol. 14: 175-186.

383 
384 16. Tomasello, M. \& Call, J. 2007. Intentional communication in nonhuman primates. In:

385 Call, J. Tomasello, M (eds) The gestural communication of apes and monkeys. Lawrence

386 Erlbaum Associates, pp 1-15.

387

388 17. Pika, S., Liebal, K. \& Tomasello, M. 2003. Gestural communication in young gorillas

389 (Gorilla gorilla): gestural repertoire, learning, and use. Am. J. Primatol. 60: 95-111.

390

391 18. Cartmill, E.A. \& Byrne, R.W. 2007 Orangutans modify their gestural signaling

392 according to their audience's comprehension. Anim. Cogn. 13: 793-804.

393

394 19. Genty, E., Breuer, T., Hobaiter, C. Byrne, R.W. 2009. Gestural communication of the

395 gorilla (Gorilla gorilla): repertoire, intentionality and possible origins. Anim. Cogn. 12: $396 \quad 527-546$.

397

398 20. Hobaiter, C. \& Byrne, R.W. 2011. The gestural repertoire of the wild chimpanzee.

399 Anim. Cogm. 14: 745-767.

400

401 21. Meguerditchian, A., Vauclair, J. \& Hopkins, W.D. 2010. Captive chimpanzees use

402 their right hand to communicate with each other: Implications for the origin of the

403 cerebral substrate for language. Cortex 46: 40-48.

404

405 22. Reynolds, V. 2005. The chimpanzees of the Budongo forest. Oxford, UK, Oxford 406 University Press. 
408 23. Altmann, J. 1974. Observational study of behavior: Sampling methods. Behaviour.

409 49(3-4): 227-266.

410

411 24. Forrester, G. S., Leavens, D. A., Quaresmini, C. \& Vallortigara, G. (2011). Target

412 animacy influences gorilla handedness. Anim. Cogn. 14: 903-907.

413

414

415 


\section{Figure captions}

418 Figure 1. Absolute hand preference index (ABS HI) for individual Sonso chimpanzees

$419(\mathrm{n}=54)$ plotted against each individual's total number of gesture instances, of the 20

420 gesture types coded for laterality. The mean across individuals, weighted by the number

421 of gesture cases, is indicated as a single line.

422

423

424 Figure 2. Hand preference index (HI) for individual Sonso chimpanzees $(n=54)$ plotted

425 against each individual's total number of gesture instances, of the 20 gesture types coded

426 for laterality.

427

428

429 Figure 3. Black bars represent mean absolute hand preference index (ABS HI) for each 430 age group, plotted on a scale of $0-1$; White bars represent mean hand preference index 431 (HI), plotted on a scale of -1 to +1 . Only individuals with 10 or more gesture instances 432 contributed data to the group mean.

435 Figure 4. Hand preference index $(\mathrm{HI})$ of individual gesture types $(\mathrm{n}=20)$, plotted against 436 the frequency of observed instances. 


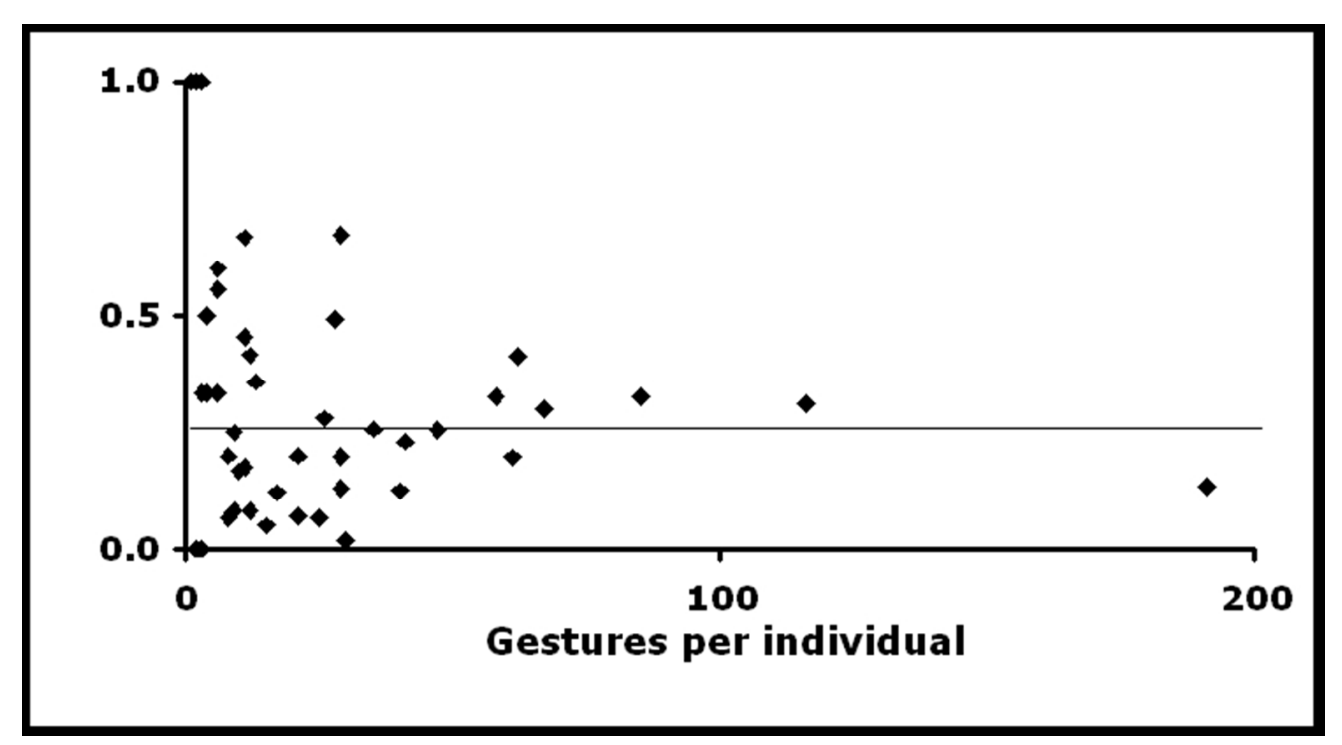

Fig 1

$196 \times 107 \mathrm{~mm}(96 \times 96$ DPI $)$ 


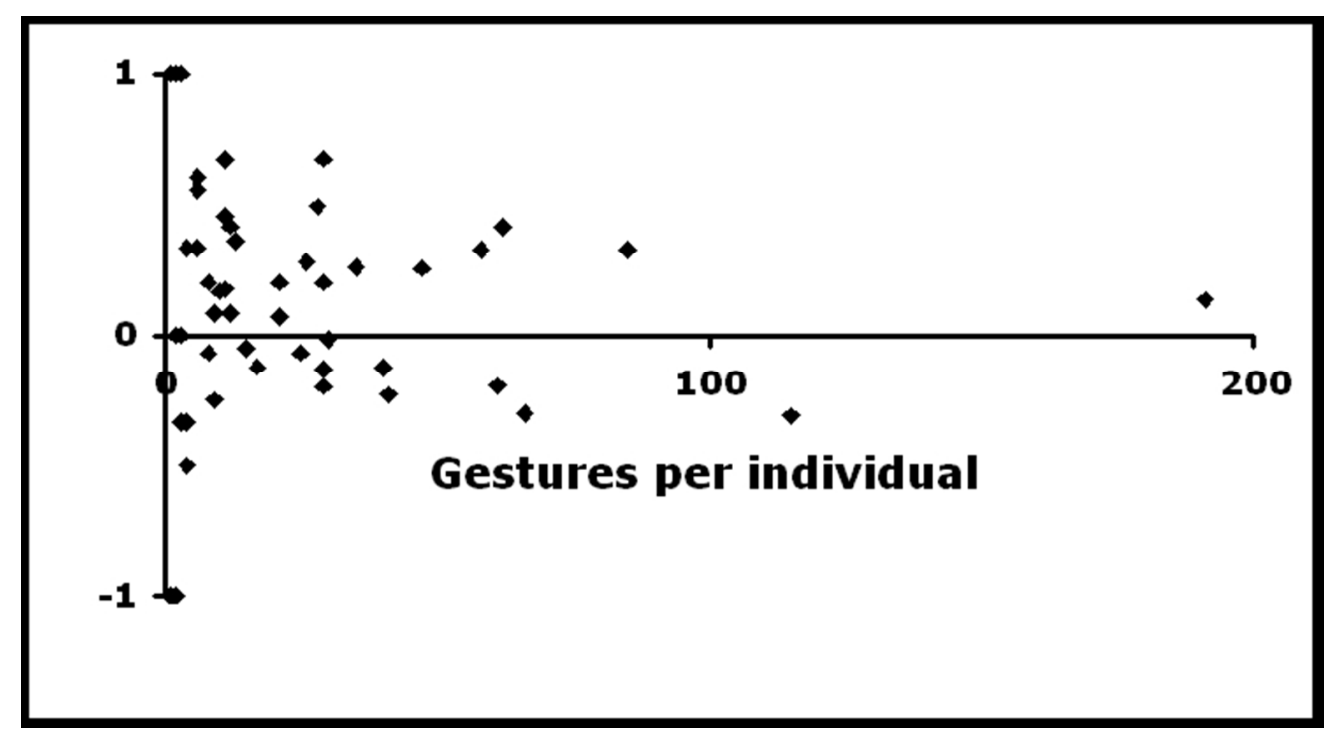

Fig 2

$196 \times 107 \mathrm{~mm}(96 \times 96$ DPI $)$ 


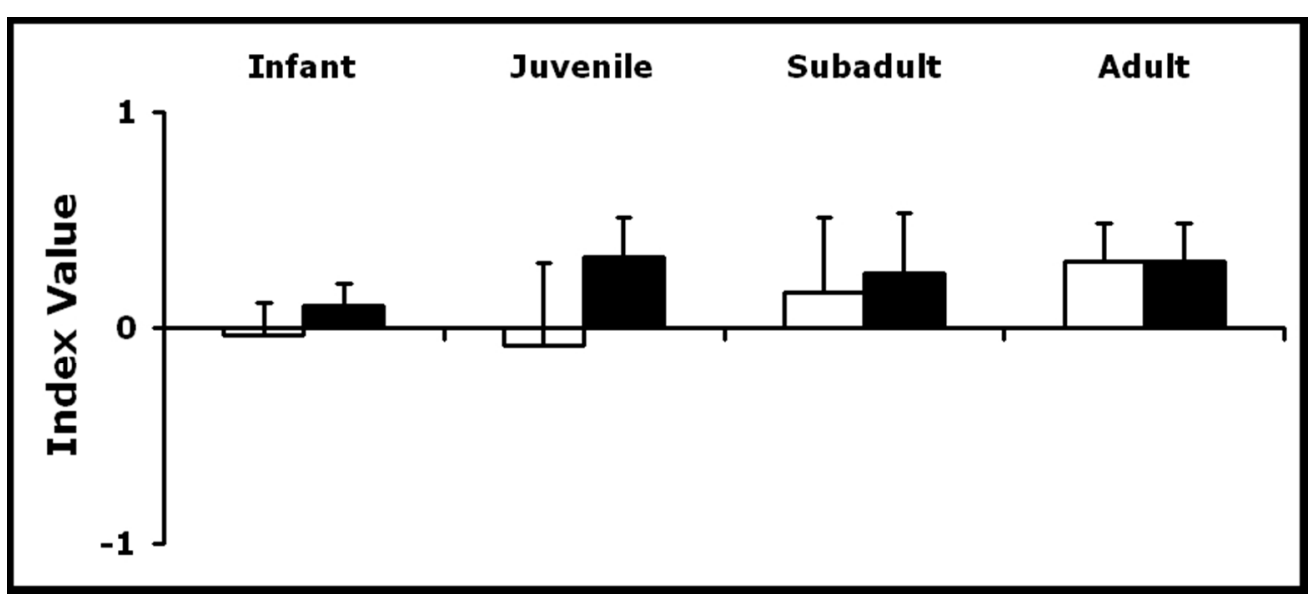

Fig 3

$236 \times 105 \mathrm{~mm}(96 \times 96 \mathrm{DPI})$ 


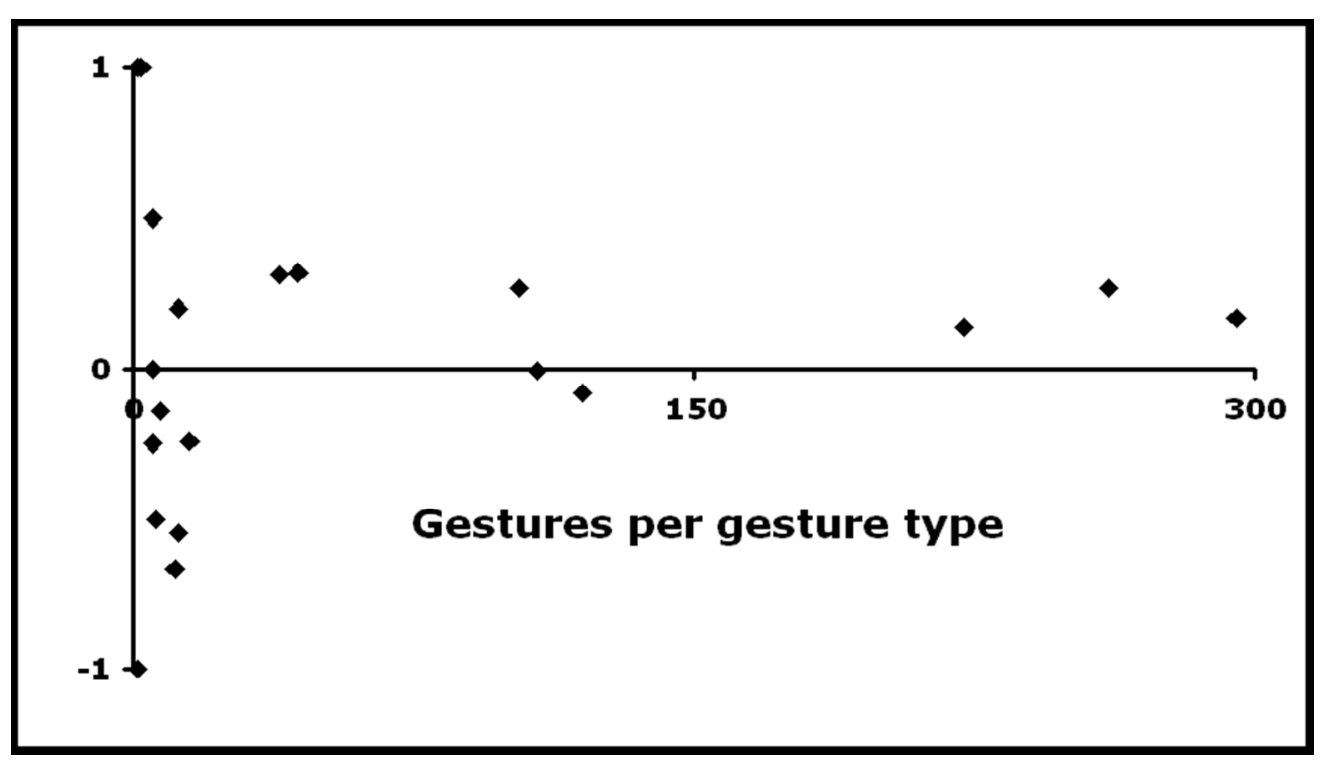

Fig 4

$228 \times 129 \mathrm{~mm}(96 \times 96 \mathrm{DPI})$ 